\title{
Evaluación del periodo de llenado y evacuación intestinal de Brachionus calyciflorus alimentado con Chlorella sp.
}

\author{
Evaluation of the period of filling and intestinal evacuation of \\ Brachionus calyciflorus fed on Chlorella sp.
}
Avaliação do período de enchimento e evacuação intestinal de Brachionus calyciflorus (Rotifera: Brachionidae) alimentado com Chlorella sp

Humberto A. Jaramillo-Campaña ${ }^{1}$, Nathalia C. Calpa-Anaguano ${ }^{2}$, Vilma Y. Gómez-Nieves ${ }^{3}$

$\begin{array}{ll}1 & \text { Ingeniero en producción Acuícola, Universidad de Nariño } \\ 2 & \text { Ingeniero en producción Acuícola, Universidad de Nariño } \\ 3 & \text { Bióloga, M Sc Unicauca-Universidad de Nariño } \\ \text { Email:humbertojara1@hotmail.com }\end{array}$

Recibido:15 de diciembre de $2018 \quad$ Aceptado:04 de junio de 2019

\begin{abstract}
Resumen
Con el fin de determinar la viabilidad del rotífero de agua dulce Brachionus calyciflorus utilizado como alimento vivo en la fase de larvicultura de especies ícticas, se evaluó el periodo cronológico de llenado y evacuación intestinal, mediante la alimentación con diferentes concentraciones de microalga Chlorella sp. en estado condensado. Los tratamientos fueron, $\mathrm{T}_{1}: 1,2 \times 10^{6}$ células $/ \mathrm{mL}, \mathrm{T}_{2}: 5,2 \times 10^{6}$ células $/ \mathrm{mL}, \mathrm{T}_{3}: 10,4 \times 10^{6}$ células $/ \mathrm{mL}$ y $\mathrm{T}_{4}: 15,4 \times 10^{6}$ células $/ \mathrm{mL}$, en una densidad de 40 rotíferos $/ \mathrm{mL}$ para cada tratamiento. Se realizó análisis de imagen, se aplicó una prueba de varianza ANOVA y test múltiple de Tukey a los resultados donde se establecieron diferencias significativas entre los tratamientos ( $\mathrm{P} \leq 0.05)$; las variables analizadas fueron: volumen del cuerpo, volumen del intestino, volumen intestinal expresado en porcentaje y tiempo de tránsito intestinal. Para el análisis morfométrico el rango de volumen del cuerpo fue de $0,7 \times 10^{6} \mu \mathrm{m}^{3}$ a $2,6 \times 10^{6} \mu \mathrm{m}^{3}$. El tiempo mínimo de llenado y evacuación fue de 60 minutos. Los porcentajes intestinales en su máxima repleción para cada tratamiento fueron $\mathrm{T}_{1}: 5,55 \%, \mathrm{~T}_{2}: 8,62 \% \mathrm{~T}_{3}: 10,53 \%$ y $\mathrm{T}_{4}: 8,08 \%$, en consecuencia, el tiempo idóneo para suministrar el rotífero B. calyciflorus como alimento vivo en larvicultura, es de 105 - 225 minutos, después de iniciado la ingesta de la microalga.
\end{abstract}

Palabras clave: Alimento vivo, microalga, rotífero, volumen intestinal.

\begin{abstract}
In order to determine the viability of the freshwater rotifer Brachionus calyciflorus used as alive food in the phase of larviculture of ictic species, there was evaluated the chronological period of filling and intestinal evacuation, by means of the supply with different concentrations of microalgae Chlorella sp. in condensed condition. The treatments were, T1: 1.2 × 106 cells / mL, T2: $5.2 \times 106$ cells / mL, T3: $10.4 \times 106$ cells / mL and T4: $15.4 \times 106$ cells / mL, all of them with a density of 40 for each treatment. Analysis of image was realized, and it was applied a test of variance ANOVA and Tukeyss multiple test to the results where significant differences between the treatments were established $(P \leq 0.05)$; the variables analyzed were: volume of body, bowel volume, intestinal volume expressed in percentage and intestinal transit time. For morphometric
\end{abstract}


analysis, the volume rank of the body was of $0,7 \times 106 \mu \mathrm{m} 3$ to $2,6 \times 106 \mu \mathrm{m} 3$. The minimum filling and evacuation time were 60 minutes. The intestinal percentages in their maximum repletion for each treatment were T1: 5.55\%, T2: 8.62\% T3: $10.53 \%$ and T4: $8.08 \%$, consequently, the suitable time to supply the rotifer B. Calyciflorus as alive food in larviculture it is of 105 - 225 minutes, after initiated the ingestion of the microalgae.

Keywords: alive food, microalgae, rotifer, intestinal volume.

\begin{abstract}
Resumo
A fim de determinar a viabilidade do rotífero de água doce Brachionus calyciflorus utilizado como alimento vivo na fase de larvicultura, foi avaliado o período cronológico de enchimento e evacuação intestinal através da alimentação com diferentes concentrações de microalga Chlorella sp. em estado condensado. Os tratamentos foram, T1: 1,2×10 ${ }^{6}$ células/ $\mathrm{mL}$, T2: $5,2 \times 10^{6}$ células $/ \mathrm{mL}, \mathrm{T} 3: 10,4 \times 10^{6}$ células $/ \mathrm{mL}$ e T4: $15,4 \times 10^{6}$ células $/ \mathrm{mL}$, numa densidade de 40 rotíferos $/ \mathrm{mL}$ para cada tratamento. Foi realizado um analise de imagem e aplicado um teste de variância ANOVA e o teste múltiplo de Tukey aos resultados dos tratamentos onde foram estabelecidas diferenças significativas ( $\mathrm{P} \leq 0.05)$; as variáveis analisadas foram: volume corporal, volume intestinal, volume intestinal expresso em porcentagem e tempo de trânsito intestinal. Para a análise morfométrica, a faixa de volume corporal foi de $0,7 \times 106 \mu \mathrm{m}^{3}$ a 2,6 ×106 $\mathrm{m}^{3}$. O tempo mínimo de enchimento e evacuação foi de 60 minutos. As porcentagens intestinais em sua repleção máxima para cada tratamento foram T1: 5,55\%, T2: 8,62\% T3: 10,53\% e T4: 8,08\%. Em consequência, o tempo ideal para fornecer o rotífero $B$. calyciflorus como alimento vivo na larvicultura é de 105 - 225 minutos, após o início da ingesta da microalga.
\end{abstract}

Palavras-chave: Comida viva, microalga, rotífero, volume intestinal.

\section{Introducción}

Los rotíferos del género Brachionus constituyen el alimento vivo de suma importancia para la primera alimentación larvaria de muchas especies de interés acuícola, debido al tamaño, velocidad de natación y capacidad para permanecer suspendido en la columna de agua, por lo que han sido ampliamente estudiados por su trascendencia para el desarrollo acuícola (Støttrup et al., 2008)

Sin embargo, el principal desafío para los científicos ha sido desarrollar una técnica de producción de rotíferos estable, sostenible, favorable al medio ambiente y de alta densidad; con el fin de satisfacer la demanda, aumentar el suministro de rotíferos, alimentar un mayor número de larvas de peces y reducir el uso de espacio para el cultivo de alimentos vivos (Dhont J., 2013)

Así mismo, la alimentación con microalgas constituye, en cualquier instalación acuícola que pretenda producir rotíferos, una técnica fundamental con grandes ventajas frente a la utilización de alimentos secos como la levadura o dietas comerciales. La utilización de microalgas genera menores desechos, reducción de impactos sobre la calidad del agua, actividad antibacteriana que disminuye la probabilidad de contaminación del cultivo (Román-reyes et al., 2014).

En cuanto a calidad nutricional potencialmente deficiente de los rotíferos como alimento para las larvas de los peces, se ha mejorado enriqueciéndolos con algunas especies de microalgas, emulsiones de aceite y productos micro particulados comerciales antes de ser suministrados a los tanques de cría (Romero et al., 2012). Así mismo, es importante resaltar que el tiempo de tránsito intestinal en rotíferos, es relativamente rápido y consecuentemente el tiempo que pasan en el tanque de cría de peces antes de ser consumido, afectará la calidad final como presa (Romero et al., 2012). En consecuencia, la supervivencia y el desarrollo normal de las larvas dependen no sólo de los nutrientes adecuados, sino también de la ingestión de alimento suficiente. Por lo tanto, el valor nutricional de los rotíferos depende de su composición bioquímica, así como de su masa seca y cantidad celular ingerida (Lubzens, et al.,1989).

Teniendo en cuenta la necesidad de conocer el tiempo de retención y la calidad nutricional que los rotíferos puedan transmitir a las larvas de peces, la presente investigación evaluó el periodo cronológico determinando el patrón de llenado y evacuación intestinal del rotífero Brachionus calyciflorus, alimentados con diferentes concentraciones de microalga Chlorella sp., por medio de un análisis morfométrico estimando: volumen intestinal con relación al volumen corporal del rotífero y determinando el tiempo de mayor retención alimenticia.

\section{Materiales y métodos}

\section{Localización}

Esta investigación se realizó en el laboratorio de Organismos Hidrobiológicos Ornamentales de la Univer- 
sidad de Nariño sede Torobajo, ubicada al noroeste de la ciudad de San Juan de Pasto, Departamento de Nariño, con una altura de 2510 msnm y temperatura promedio anual de $14^{\circ} \mathrm{C}$.

\section{Producción de Microalgas}

Para el desarrollo de esta investigación se realizó una producción escalonada de microalga Chlorella sp. (Sipabua y Rocha.,2003), partiendo del manejo de cepas en cajas Petri, tubos de ensayo, erlenmeyers de 500 $\mathrm{mL}$, recipientes de 3 y 20 litros; los cuales contenían medios de cultivo f/2 Guillard. Los cultivos se mantuvieron a una temperatura que osciló entre 22 y $24^{\circ} \mathrm{C}$, intensidad lumínica artificial 5340 Lux y aireación constante, además se utilizó glucosa a cantidades de 1g/L, como fuente de carbono orgánico (Ardila et al., 2017).

\section{Condensación de Chlorella sp.}

Obtenida la producción máxima de microalgas, se suspendió la fuente de aireación por un periodo de 8 días en presencia de luz artificial, tiempo en el cual la microalga se precipito totalmente, se cosechó y almacenó a $4{ }^{\circ} \mathrm{C}$.

\section{Cultivo de Rotíferos}

Se inició a partir de una cepa de huevos en estado de reposo (Gilbertj, n.d.), los cuales fueron depositados en unidades de eclosión (beakers $2 \mathrm{~L}$ ) con una densidad inicial de microalga $2 \times 10^{5}$ células $/ \mathrm{mL}$, temperatura constante de $28^{\circ} \mathrm{C}$ y un $\mathrm{pH}$ entre 7,5 y 8,5 . El cultivo alcanzo densidades superiores a 300 rotíferos/mL los cuales se sostuvieron con la adición de microalgas y un recambio de agua del $80 \%$ cada 72 horas.

\section{Periodo de llenado del intestino en B. calyciflorus}

Los rotíferos se mantuvieron bajo condiciones de ayuno durante 24 horas con el propósito de vaciar el intestino; se trasladaron a recipientes de vidrio de 3 litros y a densidades de 40 rotíferos $/ \mathrm{mL}$ donde se alimentaron con algas a cuatro concentraciones diferentes, $1,2 \times 10^{6} \mathrm{cel} /$ $\mathrm{mL}, 5,2 \times 10^{6} \mathrm{cel} / \mathrm{mL}, 10,4 \times 10^{6} \mathrm{cel} / \mathrm{mL}$ y $15,4 \times 10^{6} \mathrm{cel} / \mathrm{mL}$.

Para calcular la cantidad de microalga a suministrar se utilizó la fórmula de concentraciones y volúmenes:

$$
\mathrm{C}_{1} \mathrm{~V}_{1}=\mathrm{C}_{2} \mathrm{~V}_{2}
$$

La densidad sobre las unidades de cultivo fue de 40 rotíferos/mL, para evitar que la ingestión afecte la concentración de células de microalgas durante el tiempo experimental. Posteriormente, los rotíferos fueron muestreados en los cuatro tratamientos, donde se colectaron muestras cada 15 minutos durante 4 horas, para garantizar el comportamiento intestinal en presencia de alimento las muestras; se fijaron con formalina al 5\% en cajas multiceldas, se llevaron a microscopio y se tomaron fotografías, donde se midió el área del cuerpo y del intestino por medio del software Image J, como se explica en la parte de análisis morfométrico.

\section{Patrón de evacuación intestinal en B. calyciflorus}

Para determinar el patrón de evacuación, los rotíferos fueron alimentados primero con microalgas a diferentes concentraciones según los tratamientos mencionados anteriormente. A continuación, se eliminaron las células de microalgas por filtración húmeda, la cual consistió en descartar las células conservando rotíferos en un volumen de agua reducido. Los rotíferos se muestrearon en diferentes momentos durante un periodo de 3 horas (debido a que el periodo de evacuación intestinal es menor al periodo de llenado intestinal) y se fijaron en formalina al 5\% en cajas multiceldas, la primera muestra se tomó después de la filtración húmeda (considerada como tiempo 0) a partir del cual se tomaron muestras consecutivas cada 15 minutos, se llevaron a microscopio y se tomaron fotografías sucesivamente. La disminución del volumen del intestino se presentó como porcentaje del volumen total del cuerpo.

\section{Análisis morfométrico}

Se realizó un análisis de imagen en el 20\% de la población de cada muestra, se recolecto información fotográfica mediante microscopia directa, las imágenes fueron procesadas utilizando el software libre Image J; el ancho, la longitud del cuerpo y el área del intestino se midieron en cada individuo. Para la medida del intestino el criterio fue considerar sólo el área de las vesículas que contenían microalgas, esta zona estuvo compuesta de 1 o 2 manchas correspondientes a las cavidades intestinales.

El volumen del cuerpo del rotífero se calculó de acuerdo con un elipsoide de revolución, donde se obtuvieron el ancho y la longitud $(\mu \mathrm{m})$ midiendo la lorica sin espinas para cada individuo (Yufera, 1982).

$$
\text { Volumen de rotiffero }\left(\mu m^{3}\right)=\frac{4}{3} \pi \times a \times b^{2}
$$

Dónde:

a y b son la mitad de los ejes mayor y menor (longitud y anchura), respectivamente. 


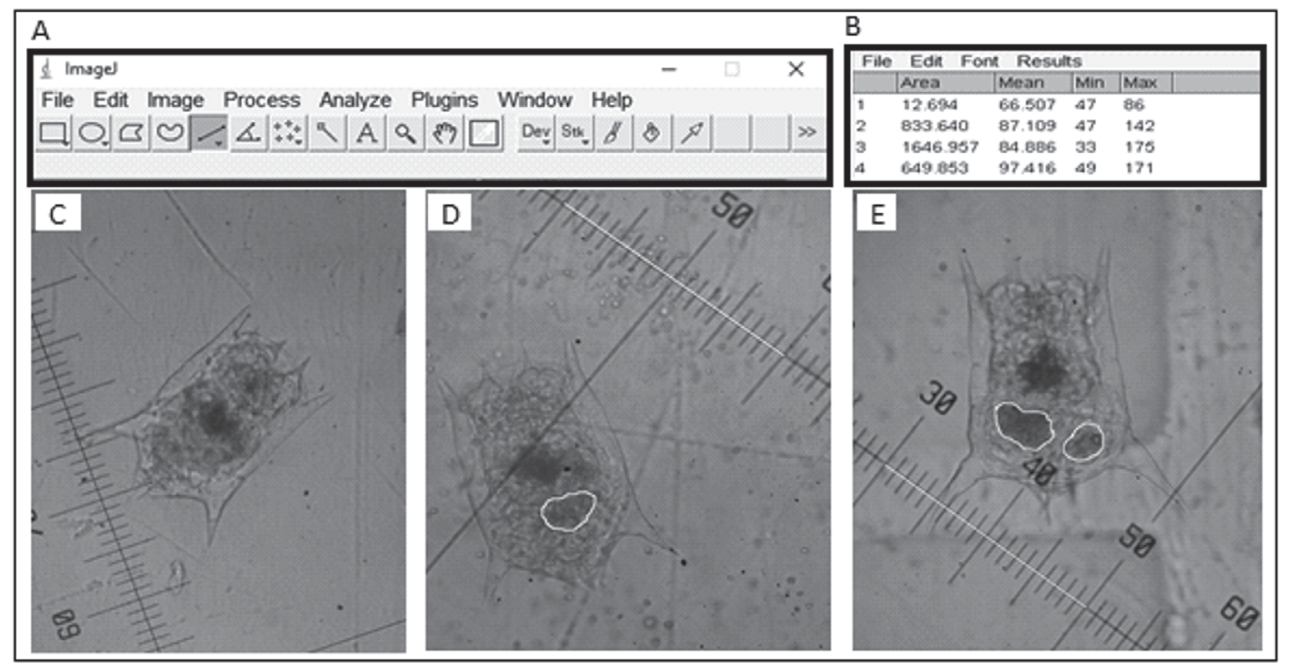

Figura 1. Análisis de imagen, A. Barra de herramientas ImageJ, B. Cuadro de diálogo con áreas, C. Rotífero vacío, D. Rotífero con una cavidad intestinal, E. Rotífero con doble cavidad intestinal (Fuente: esta investigación).

El volumen del intestino se calculó mediante la obtención de áreas (Romero et al., 2012). Se considera que las cavidades intestinales tienen una forma esférica y el volumen se calculó a partir del área.

$$
\text { Volumenintestinal }\left(\mu m^{3}\right)=\frac{4}{3}\left(A_{t} \sqrt{\frac{A_{1}}{\pi}}\right)+\frac{4}{3}\left(A_{2} \sqrt{\frac{A_{2}}{\pi}}\right)
$$

Dónde:

$\mathrm{A}_{1}$ y $\mathrm{A}_{2}$ corresponden al área $\left(\mu \mathrm{m}^{2}\right)$ de las dos cavidades intestinales medida en las imágenes.

Teniendo en cuenta la variabilidad del tamaño corporal, el volumen del intestino se presentó como porcentaje relacionado con el volumen total del cuerpo.

$$
\% \text { intestinal }=\frac{V_{1}}{V_{c}} \times 100
$$

Dónde:

$V_{\text {I }}$ es el volumen del intestino, $V_{C}$ es el volumen del cuerpo del rotífero.

Para el tiempo de llenado y evacuación intestinal se calculó teniendo en cuenta el tiempo inicial y final de la investigación.

$$
T=T_{F}-T_{l}
$$

Dónde:

$T_{F}$ es el tiempo en el que se estabiliza la curva de crecimiento en el tránsito intestinal y $\mathrm{T}_{\imath}$ es el tiempo en el que inicia el estudio.

\section{Análisis estadístico}

Se realizaron modelos lineales, con el fin de verificar si existía relación entre el volumen del rotífero, el tratamiento, el tránsito intestinal y el tiempo en que se ejecutó la investigación. Se aplicó análisis de varianza (ANOVA), al encontrar diferencias significativas entre tratamientos (aplicando logaritmo +1 a la variable volumen intestinal) se realizó una prueba múltiple de Tukey para establecer la diferencia significativa entre tratamientos. Todas las pruebas se efectuaron con $\mathrm{P}<0.05$ y todos los datos se analizaron a través del programa estadístico $\mathrm{R}$.

\section{Resultados y discusión}

El análisis morfométrico se realizó con base al volumen corporal del rotífero, para este caso la prueba ANOVA con nivel de significancia $(P \leq 0.05)$, estableció diferencias significativas entre los tratamientos, así mismo el test de Tukey mostro diferencias estadísticas entre tratamiento.

El rango de volumen del cuerpo osciló entre $0,7 \times 10^{6}$ $\mu \mathrm{m}^{3}$ a $2,6 \times 10^{6} \mu \mathrm{m}^{3}$, encontrándose más del $90 \%$ de la población entre un volumen corporal de $1 \times 10^{6}$ y 2,5 $\times 10^{6} \mu \mathrm{m}^{3}$ (Figura 2).

Xue et al., (2017) en un estudio en rotíferos B. calyciflorus encontraron un rango de $1,6 \times 10^{6}$ a $2,2 \times 10^{6} \mu^{3}$, ajustándose a los datos evaluados en esta investigación. En el caso de rotíferos Brachionus plicatilis, los datos reportados sobre volumen del cuerpo oscilaron entre 0,73 y $4,21 \times 10^{6}{\mu m^{3}}^{3}$ situando la mayoría de la población entre $1,5 \times 10^{6} \mu \mathrm{m}^{3}$ y $2 \times 10^{6} \mu \mathrm{m}^{3}$ (Romero et 


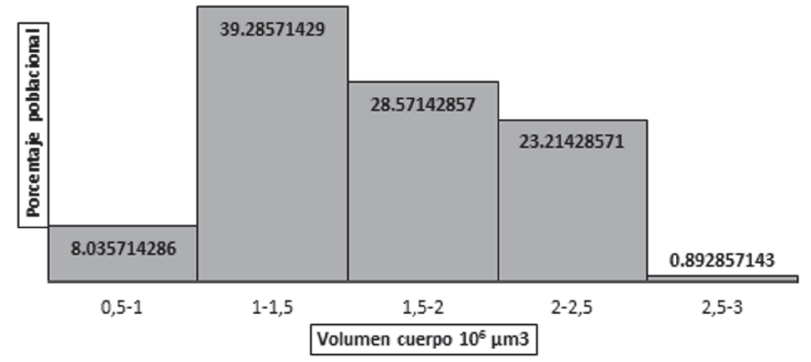

Figura 2. Histograma de frecuencia para volumen del cuerpo.

al., 2012), Por otra parte, el volumen corporal promedio en rotíferos del género Brachionus es de 1,51×106 $\mu^{3}$ (Baer, Langdon, Mills, Schulz, y Hamre, 2008) el cual fue similar al valor promedio obtenido en este estudio siendo este $1,6 \times 10^{6} \mu^{3}$.

\section{Tiempo de llenado y evacuación intestinal}

Para el tiempo de llenado se tuvo en cuenta las variables porcentaje intestinal y tiempo en la primera fase de la investigación. En la Figura 3 se muestra las curvas de regresión lineal que indicaron la velocidad de llenado intestinal hasta los 240 minutos de estudio. Los rotíferos iniciaron el periodo de llenado inmediatamente después de suministrar las concentraciones de microalga correspondiente a cada tratamiento. A los 60 minutos los rotíferos Ilenaron una parte considerable de su intestino, aproximadamente un $80 \%$ del contenido intestinal. Estos valores fueron similares a los mencionados por Romero et al., (2012), quienes reportan un intestino Ileno 60 minutos después del inicio de la alimentación. Baer et al., (2008) en un experimento con Brachionus sp. "Cayman" utilizo micro capsulas de látex reportando un periodo de llenado más corto de 35 minutos después de iniciado el enriquecimiento o alimentación, pero ese volumen aumentó ligeramente después de 20 minutos adicionales manteniéndose estable durante el resto del estudio, lo cual se asemeja al tiempo de llenado observado en esta investigación.

En la Tabla 1 se observa el tiempo en que se estabilizó la curva de regresión lineal con los volúmenes intestinales para cada tratamiento. Después de este periodo se observó una leve variación en el volumen intestinal durante el resto del estudio aclarando que esa variación fluctuó entre el 1\% y $2 \%$.

Para el tiempo de evacuación intestinal se tuvo en cuenta las variables porcentaje intestinal y tiempo en la segunda fase de la investigación. En la Figura 4, se

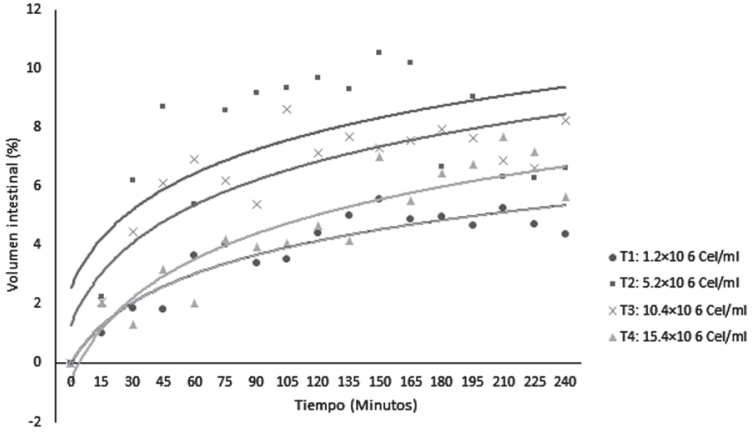

Figura 3. Periodo de Llenado intestinal del rotífero Brachionus calyciflorus en los tratamientos.

Tabla 1. Tiempo de estabilización fase de llenado

\begin{tabular}{|c|c|c|c|c|}
\hline $\begin{array}{c}\text { Tratamiento } \\
(\text { Cel } / \mathbf{m L})\end{array}$ & $\mathbf{1 , 2}^{2 \times \mathbf{1 0}^{\mathbf{6}}}$ & $\mathbf{5 , 2 \times \mathbf { 1 0 } ^ { \mathbf { 6 } }}$ & $\mathbf{\mathbf { 1 0 } ^ { 2 } \mathbf { 4 } \times \mathbf { 1 0 } ^ { \mathbf { 6 } }}$ & $\mathbf{1 5 , 4 \times \mathbf { 1 0 } ^ { \mathbf { 6 } }}$ \\
\hline Vol. intestinal & 4,06 & 6,22 & 8,59 & 4,19 \\
\hline Tiempo (min) & 60 & 60 & 60 & 60 \\
\hline
\end{tabular}

expresan las curvas de regresión que indican la velocidad de evacuación.

Los datos expresados para el proceso de evacuación indicaron que a los 60 minutos de eliminadas las concentraciones celulares se produjo la evacuación intestinal parcial $(70 \%$ del contenido intestinal del rotífero B. calyciflorus).

En el caso de Brachionus sp. "Cayman" el proceso de evacuación oscilo entre 20 y 30 minutos (Baer et al., 2008), sin embargo Romero et al., (2012) afirma que la disminución del volumen del intestino se produce progresivamente y se necesita 60 minutos para perder el $60 \%$ del contenido, además sostiene que las curvas de evacuación son similares cuando los rotíferos se re-suspenden sobre agua limpia, como se obtuvo en esta investigación a excepción del $\mathrm{T}_{1}$ que presentó un proceso de evacuación más lento a comparación de los demás tratamientos debido a la baja cantidad de

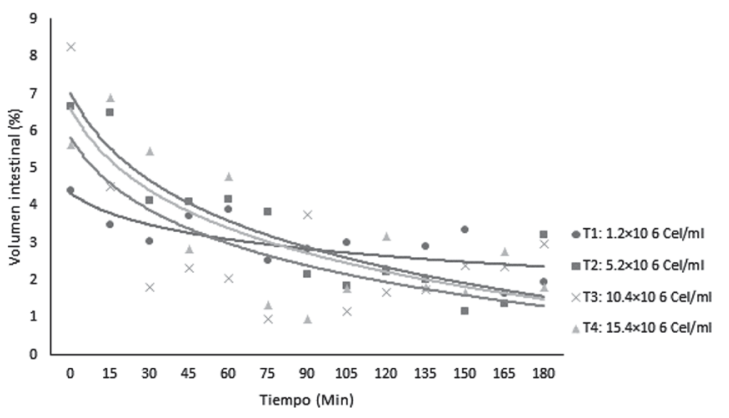

Figura 4. Periodo de Evacuación intestinal del rotífero Brachionus calyciflorus para los diferentes tratamientos. 
alga suministrada (Romero et al., 2012). Incluso Dhert et al., (2001) afirman que la evacuación intestinal se producirá de manera más lenta en un sistema de agua verde con $0.4 \times 10^{6} \mathrm{cel} / \mathrm{mL}$. En el caso de enriquecimiento con yodo y vitaminas en rotíferos Brachionus $s p$, la evacuación se produjo después de 60 minutos (Srivastava et al., 2012), resultado similar al obtenido en este estudio.

\section{Volumen intestinal en su máxima repleción en relación con el volumen corporal del rotífero}

El volumen máximo obtenido fue de 201.752,09 $\mu \mathrm{m}^{3}$ que representó el $10.53 \%$ del volumen total del cuerpo, estos porcentajes fueron relativamente bajos en relación a los encontrados por Romero et al., (2012) de $13 \%$ en su máxima repleción. Otros investigadores como Kleinow et al., (1991) establecieron que el volumen intestinal alcanza las $200.000 \mu^{3}$. Teniendo en cuenta los valores máximos obtenidos en esta investigación, se puede afirmar que el volumen del cuerpo en relación con el porcentaje intestinal se vio afectado por la concentración celular y el tamaño del rotífero, no obstante, el volumen intestinal no se vio afectado por el tamaño del rotífero; reportándose porcentajes intestinales similares independientemente del tamaño del individuo (Tabla 2).

El tratamiento 4 presentó volúmenes inferiores con respecto al tratamiento 2 y 3 (Tabla 2) por lo tanto; una menor ingestión celular. Yufera et al., (1985), en un estudio con Brachionus plicatilis afirmó que las tasas de ingestión disminuyen a concentraciones de células superiores a $15 \times 10^{6} \mathrm{Cel} / \mathrm{mL}$.

Romero et al., (2012), en Brachionus plicatilis reportó volúmenes intestinales estables entre 60 y 180 minutos, entre los 210 y 225 minutos experimentaron un leve descenso antes de la filtración húmeda. A pesar de que los volúmenes máximos intestinales para esta investigación se mantuvieron estables, fluctuaron con mayor frecuencia cerca de los 215 minutos.
Para determinar el tiempo de viabilidad del rotífero se tuvo en cuenta el mejor tratamiento, en este caso el tratamiento 2 con una concentración de 5,2×106 células $/ \mathrm{mL}$, donde el tiempo de retención intestinal se encontró entre un rango de 105 - 225 minutos, tiempo en el cual los volúmenes intestinales se mantuvieron estables. Romero et al., (2012) encontraron que el tiempo óptimo de enriquecimiento osciló entre 120 y 180 minutos, además, deduce que un periodo de alimentación más largo conduce a una disminución en el volumen intestinal del rotífero, por otra parte, Baer et al., (2008) también encontró el mismo fenómeno y deduce que es la consecuencia del inicio de la evacuación después de un tiempo de retención intestinal probablemente debido a un efecto de rebote.

\section{Agradecimientos}

A la Universidad de Nariño que permitió el desarrollo de esta investigación en sus instalaciones, al Ecólogo Carlos Trujillo, a los Biólogos Edna Calpa, Jullbrainer Salas y a todas las personas que colaboraron en la realización de esta investigación.

\section{Bibliografía}

Ardila A, López Y, Vásquez M, Gonzales A, Barajas A. Obtención de lípidos y carbohidratos a partir de microalgas mediante el diseño de medios de cultivo selectivos. Tecno Logicas. 2017;20:8394.

Baer A, Langdon C, Mills S, Schulz C, Hamre K. Particle size preference, gut filling and evacuation rates of the rotifer Brachionus "Cayman" using polystyrene latex beads. En Aquaculture. 2008;282(1-4):75-82.

Dhert P, Rombaut G, Suantika G, Sorgeloos P. Advancement of rotifer culture and manipulation techniques in Europe. Aquaculture. 2001:130-146.

Dhont In G. Allen (Ed.). En Advances in Aquaculture Hatchery Technology. Cambridge: Woodhead Publishing. Series in Food Science, Technology and Nutrition; 2013;242:167-191. J. Rotifers, Artemia and copepods as live feeds for fish larvae in aquaculture.

Tabla 2. Valores máximos alcanzados en la investigación.

\begin{tabular}{|c|c|c|c|}
\hline Tratamiento & Vol. Cuerpo $\boldsymbol{\mu m}^{\mathbf{3}}$ & Vol. Intestinal $\boldsymbol{\mu m}^{\mathbf{3}}$ & \% Intestinal \\
\hline 1 & $2.255 .212,67$ & $87.683,64$ & 5,55 \\
\hline 2 & $1.695 .434,45$ & $107.451,90$ & 8,62 \\
\hline 3 & $2.632 .882,57$ & $201.752,09$ & 10,53 \\
\hline 4 & $1.986 .483,05$ & $100.636,10$ & 8,08 \\
\hline
\end{tabular}


Gilbertj JJ. (n.d.). Mictic Female Production in the Rotifer Brachionus calyciflorus, 113-123.

Kleinow W, Wratil H, Kuhle K, Esch B. 1991. Electron microscope studies of the digestive tract of Brachionus plicatilis (Rotífera). En Zoomorphology. p 67-80

Lubzens E, Tandler A, Minkoff G. Rotifers as food in aquaculture. Hydrobiologia. 1989;186(1):387-400.

Román J, Castañeda D, Castillo H, Bojórquez R, Rodríguez G. Dinámica poblacional del rotífero Brachionus ibericus aislado de estanques para camarón, alimentado con diferentes dietas. Latin american journal of aquatic research, 2014;42(5):1159-1168.

Romero S, Yúfera M. Contribution of gut content to the nutritional value of Brachionus plicatilis used as prey in larviculture. En Aquaculture. 2012;364:124-129.

Sipabua L, Rocha O. 2003. Produção de plâncton (Fitoplâncton e Zooplâncton) para a alimentação de organismos aquáticos. RiMa, São Carlos.
Støttrup J, McEvoy L. 2008. Live feeds in marine aquaculture. Vol. 1 st ed. Oxford, Oxford: John Wiley \& Sons. Blackwell Science. p. 17-52.

Srivastava A, Hamre K, Stoss J, Nordgreen A. A study on enrichment of the rotifer Brachionus "Cayman" with iodine from different sources. Aquaculture. 2012;334:82-88.

Xue Y, Yang X, Zhang G, Xi Y. Morfological differentiation of Brachionus calyciflorus caused by predation and coal ash pollution. En Scientific reports. 2017;7(1):1-8.

Yufera M. Morphometric characterization of a small-sized strain of Brachionus plicatilis in culture. Aquaculture. 1982;27(1):55-61.

Yúfera M, Pascual E. Effects of algal food concentration on feeding and ingestion rates of Brachionus plicatilis in mass culture. Hydrobiologia. 1985;122(2):181-187. 